\title{
Pfarrbrief statt Bistumsblatt Plädoyer für eine neue Medienpolitik der Kirche in den Niederlanden
}

\author{
von Joan Hemels
}

\section{Thematische Einleitung}

Ein spezifisches Kennzeichen der Presselandschaft innerhalb der katholischen Kirche der Niederlande ist die Dualität von Bistumsblättern und Pfarrbriefen. An den Bistumsblättern besitzen jeweils die Ortsbischöfe die Eigentumsrechte. Daneben haben die Pfarrgemeinden in den letzten Jahrzehnten ihre kirchlichen Berichte in die Pfarrblätter eingebracht, die die Grundlage der Kommunikationsstruktur der katholischen Kirchengemeinden bilden. Der vorliegende Beitrag beschäftigt sich in vergleichender Absicht mit diesen beiden Formen katholischer Printmedien und plädiert für eine stärkere Gewichtung des Pfarrbriefs. Zugleich werden grundsätzliche Fragen nach der Relevanz der heutigen Bistumsblätter der katholischen Kirche der Niederlande aufgeworfen. ${ }^{1}$

Für die Meinungsbildung über das Funktionieren von Medien, die im Besitz der Kirche sind, ist der Wandel im kirchlichen Denken zwischen dem Konzilsdekret 'Inter Mirifica' (1963) und der Pastoralinstruktion 'Communio et progressio' (1971) noch immer von großer Bedeutung. Das instrumentalistische Denken, das die Verwendung der Massenmedien in den Dienst der Kirche und ihrer Interessen stellt, ist typisch für die Zeit vor 'Inter mirifica'. In 'Communio et progressio' wird dagegen erstmals der Wert der Kommunikation 'um der Kommunikation willen' und nicht 'als etwas instrumentalistisches' anerkannt. So bekam auch die Massenkommunikation als Auftrag der Kirche eine neue Bedeutung und einen neuen Inhalt. Es ging jetzt nicht mehr ausschließlich um den Dienst an einer - meist hierarchiekonformen und abgegrenzten Aufgabe,also um eine instrumentalistische Funktion, sondern um eine Kommunikation ${ }_{n}$ um des Dialogs selbst willen“. ${ }^{2}$

Zwischen dem Geist der Pastoralinstruktion und der alltäglichen Praxis kann 20 Jahre später in manchen Fällen noch eine gefährliche Schlucht liegen. Dies wurde u. a. im Sommer 1991 deutlich. In manchen Presseorganen, darunter auch im Bisdomblad ('s Hertogensbosch) vom 9. August 1991, konnte man von einer Entlassungsaktion in Augsburg lesen. Der Chefredakteur des Ulrichblattes, der Kirchenzeitung für die Diözese Augsburg, Pater Gerhard Eberts m.s.f., wurde von seinem Bischof entlassen. Über die wirklichen und die angegebenen Gründe dieser Entlassung gab es unterschiedliche Berichte. Nur soviel scheint deutlich zu sein: daß der Bischof mit seinem Chefredakteur nicht mehr durch die gleiche Kirchentür gehen konnte.

Prof. Dr. Joan Hemels ist Inhaber des Lehrstuhls für Kommunikationswissenschaft, insbesondere Kommunikationsgeschichte, der Studienrichtung Kommunikationswissenschaft der Universität Amsterdam, Niederlande. 
In den Niederlanden entstanden im Herbst 1991 Gerüchte über Unstimmigkeiten zwischen dem Bischof von Haarlem, H. Bomers, und Dr. Johan Hahn, Dozent an der katholisch-theologischen Universität Amsterdam und nebenberuflichem Redakteur von 'Samen Kerk', dem Haarlemschen Bistumsblatt. Hahn sah sich gezwungen, die Redaktion von 'Samen Kerk' aufzugeben, um die Chance auf Fortsetzung seiner Dozentur in Kommunikationswissenschaften bei der am 1. Januar 1992 durchgeführten Fusion der Amsterdamer katholisch-theologischen Universität mit der katholisch-theologischen Universität Utrecht nicht zu gefährden. ${ }^{3}$

Die neueste Entwicklung im Bistum Haarlem bestätigt die schon seit Jahren bestehenden Spannungen zwischen dem konservativen Bischof Bomers und der Redaktion des Bistumsblatts 'Samen Kerk' und tut dem Ansehen der Amtskirche Abbruch. Der Bischof versuchte 1991 ohne Erfolg, eine neue, konservativ eingestellte Redakteurin zu ernennen, die von der Redaktion nicht gewünscht wurde. Im Frühjahr 1992 bekam der Bischof von Privatleuten ein alternatives Bistumsblatt geschenkt, mit der verschmähten Kandidatin als Chefredakteurin und finanziert aus Privatmitteln. Die inhaltliche Linie dieser 'De Apostel' getauften Zeitschrift (nomen est omen!) wird umschrieben als 'im Einklang mit der universalen und allgemeinen Lehre der Kirche' stehend. Bomers hat seine Mitarbeit an 'De Apostel', das der offiziellen Lehre der Kirche mehr Aufmerksamkeit widmen wird, schon zugesagt.

Die hierarchische Kommunikationsstruktur der Kirche hat in der Zeit zwischen 'Inter Mirifica' und 'Communio et progressio' durch gesellschaftliche Veränderungen viel von ihrer Selbstverständlichkeit verloren. Internationale und nationale Unternehmen, Universitäten, private Organisationen und andere. Einrichtungen haben sich systematisch auf Prozesse und Strukturen interner Kommunikation besonnen. Daraus entwickelten sich Strukturen der Mitsprache und Mitbeteiligung. Auch wurden Kommunikationstechniken und Medien eingesetzt, um die Menschen zu informieren und zu motivieren, die bei solchen Organisationen arbeiteten. An der niederländischen nachkonziliaren Kirche sind diese Veränderungen nicht vorbeigegangen. Bezeichnend scheint mir in diesem Zusammenhang zu sein, daß der Begriff 'kirchlich sprechen' aus dem Bewußtsein verschwunden ist. Dadurch entstand Raum für 'kirchlich zuhören'.

Die Ernennung von Frans Oudejans 1963 zum ersten Pressesprecher eines niederländischen Bischofs im Bistum Breda darf denn auch mit einer neuen Periode in Zusammenhang gebracht werden: mit der Professionalisierung des 'In-die-Öffentlichkeit-Tretens' der Kirche und ihrer Leiter. 1972 erhielt die niederländische Kirche eine eigene Abteilung für Presse und Öffentlichkeitsarbeit. Es läßt sich vermuten, daß die Bistümer in diesem Zusammenhang wieder verstärkt Interesse an einer regelmäßigen Publikation bekamen, um den Kontakt zwischen den Katholiken und dem Bistum zu verstärken. Oder sollte eher gesprochen werden von der Notwendigkeit eines Kontaktes des Bistums mit der Basis?

Jedenfalls ist die Tatsache, daß nicht alle Bistumsblätter der 70er und 80er Jahre zur vollen Entfaltung kamen, ein Grund für eine Besinnung. Meiner Ansicht nach ist eine durchgreifende und schnelle Reorganisation unvermeidlich. 
Um diesen Standpunkt akzeptabel zu machen, gehe ich zunächst auf Umfeldfaktoren ein, mit denen die Bistumsblätter besonders in den Niederlanden konfrontiert werden. Danach versuche ich deutlich zu machen, daß es in der niederländischen Situation sehr wünschenswert ist, den Pfarrblättern Priorität zu geben. Zum Schluß versuche ich einen Plan zu entwickeln, der auf die Aufhebung aller bestehenden Bistumsblätter abzielt. Auf diese Weise wäre der Weg frei für die Entwicklung eines neuen, wöchentlich erscheinenden unabhängigen Magazins für die Katholiken der verschiedensten Richtungen in der niederländischen Kirchenprovinz. Falls finanziell machbar, sollte die Möglichkeit nicht ausgeschlossen werden, solch ein neues Blatt mit Wechselseiten oder Wechselbögen für jedes Bistum zu versehen.

\section{Das Auf und $\mathrm{Ab}$ in der Entwicklung}

Der Wandel von einer in Jahrhunderten gewachsenen, geschlossenen und stark hierarchischen Kommunikationskultur zu einer offenen dialogischen Kommunikationskultur ist offenbar nicht während einer Generation zu verwirklichen. Betrachtet man die niederländische Situation, so könnte man selbst von Rückentwicklungen sprechen. Es ist nur ein schwacher Trost zu wissen, daß das "Volk Gottes unterwegs" inzwischen auch in anderen Ländern mit Bischofsernennungen konfrontiert wird, die als eine Bedrohung des Geistes des II. Vatikanischen Konzils erfahren werden. Gerade in solchen Augenblicken wird man sich der hierarchischen Struktur und ebenso einer hierarchischen Kommunikationskultur peinlich bewußt. Die Niederlande, Österreich, die Schweiz und der wallonische Teil Belgiens liefern Beispiele von Bischofsernennungen, die in den Medien zu einer langdauernden Aufregung führten. Die schon erwähnten Aktivitäten sollten zur Verminderung solcher negativen Publizität mit Kommunikationsplänen zur Verbesserung der externen Kommunikation ins Rennen gehen.

Bei einem Rückblick auf 20 Jahre 'Communio et progressio' auf einem internationalen Kongreß im Juni 1991 in Wien hat der Münsteraner Fundamentaltheologe Johann Baptist Metz die kirchliche Kommunikationskultur seit dem II. Vatikanischen Konzil kritisch untersucht. Er legte den Finger auf den eurozentrischen Charakter der modernen Massenmedien und machte darauf aufmerksam, daß die 'sozial geteilte und kulturell polyzentrische Weltkirche' in der Gefahr stehe, sich mit einer eurozentrischen Medienkultur zu identifizieren. Die Kirche, die so viel Zeit benötigte, um die Bedeutung von Kommunikationsprozessen in ihren eigenen Reihen und auch 'draußen' zu erkennen, droht nach Metz in einen neuen Fallstrick zu geraten: nämlich den Fallstrick einer Kommunikationskultur, die ihre Wurzeln in einer 'rationalen und kommerziellen Machtkultur' besitzt, mit unzureichendem Respekt vor der Freiheit und der Würde der anderen. ${ }^{4}$ Eine unkritische Identifikation mit dieser eurozentrischen Kommunikationsstruktur und -kultur würde die Glaubwürdigkeit der Kirche noch weiter unterminieren.

Bei der Behandlung der bestehenden und eventuell zu ändernden Situation der Bistumsblätter in der niederländischen Kirchenprovinz kann 'Commu- 
nio et progressio' als die 'Magna charta' der kirchlichen Medien-Aktivitätens nicht unberücksichtigt bleiben. In den Niederlanden ist dieses Dokument viel zu wenig diskutiert und bedacht worden. Und ob wir davon lernen wollen, ist auch nicht so klar. Manchmal liest man Texte, die den Eindruck erwecken, als ob diese pastorale Instruktion selbst im Arbeitszimmer des einen oder anderen Bischofs unter einer Staubschicht liege. Ein Beispiel dazu reicht m. E. aus, um dieses Gefühl der Enttäuschung über den Einfluß von 'Communio et progressio' in der niederländischen Kirchenprovinz zu schildern.

In der Rubrik 'Wort des Bischofs' im Bisdomblad vom 11. Januar 1991 schreibt der Bischof von 's-Hertogenbosch, J. ter Schure, in einer Betrachtung über 'Die öffentliche Meinung' gleichsam als Schlußfolgerung: 'Die öffentliche Meinung, auch in der Kirche, ist ein Monstrum, daß seine eigenen Kinder verschlingt. ${ }^{6}$

Tendenz und Schlußfolgerung dieses bischöflichen Wortes stehen im Gegensatz zu Buchstaben und Geist von 'Communio et progressio' und datieren aus einer Zeit, die weit vor dem II. Vatikanischen Konzil liegt.

Welch eine Erleichterung bedeuten dann die Worte, die der flämische Medienbischof, L. de Hovre, an die Teilnehmer des Interdiözesanen Pastoralen Rates gerichtet hat. Dieses höchste Beratungs- und Mitverantwortungsorgan der flämischen Kirchengemeinschaft hat sich am Ende der achtziger Jahre sehr eindringlich mit den Beziehungen zwischen den Christen und den Medien beschäftigt. ${ }^{7}$ Bischof De Hovre hat sich in seinen Worten sehr intensiv auf 'Communio et progressio' bezogen und das Interesse einer kirchlichen Präsentation in den Medien aus den folgenden vier Blickwinkeln besprochen:

- die Medien als Mittel zur Verkündigung des Evangeliums;

- die Mitwirkung am Kommunikationsprozeß in der Gesellschaft;

- die Präsenz in einem neuen, wichtigen Teil unserer Kultur, sowie

- Stimme derer sein, die keine Stimme haben. ${ }^{8}$

Diese Zielfelderbestimmung könnte eine Quelle der Inspiration sein für diejenigen, die sich mit kirchlichen Medienaktivitäten in den Niederlanden beschäftigen.

Die Prozesse der Säkularisierung und Entkonfessionalisierung, mit denen sich die Kirche seit Jahrzehnten konfrontiert sieht, äußern sich nicht nur in einem Rückzug der Kirchen aus solchen Gebieten, die sie vorher zu ihrer 'Einflußsphäre' rechnen durften. Außer einem sozialstrukturellen Prozeß hat Säkularisierung auch eine subjektive Komponente. Säkularisierung der Gesellschaft und der Kultur ist an eine Säkularisierung des Bewußtseins gekoppelt. Dies bedeutet, daß die Menschen immer mehr auf 'die Welt' schauen, ohne sich religiöser Interpretationen zu bedienen.

Warum viele katholische Zeitungen und Publikumszeitschriften im Prozeß der Säkularisierung so einfach "mitglitten“ bzw. ihren Lesern nicht so sehr folgten, sondern ihnen vielfach sogar "vorausgingen“, ist noch nicht klar. Möglicherweise handelte es sich.dabei um eine Gegenreaktion auf eine stark von der 
Hierarchie vorgegebene Aufgabenzuweisung an die Journalisten der Kirchenpresse. Es kann auch sein, daß die Verleger oder Redaktionen fürchteten, den Kontakt mit ihren Lesern zu verlieren, die offensichtlich von einer Zeitung oder Zeitschrift immer weniger 'weltanschauliche Orientierung' erwarteten. Die Richtungsblätter im allgemeinen gerieten in den 60er Jahren in Schwierigkeiten, weil sie in bestimmten Marktsegmenten keine Führungsposition behalten konnten. Wer kein Fremder in Jerusalem ist, der weiß aus persönlicher Erfahrung, daß Interesse für Religion und geistliches Leben sozial nicht besonders akzeptiert und angesehen ist. Denkt man an E. Noelle-Neumanns Theorem von der 'Schweigespirale', ist es durchaus vorstellbar, daß Antworten auf bestimmte Fragen z. B. bei Leserumfragen, von dieser Mentalität beeinflußt werden. Das Gespenst der schweigenden Mehrheit wäre dann nicht nur in politischen, sondern auch in religiösen Fragen aktiv.

\section{Kontext und Nährboden eines Bistumsblattes}

Über Ort und Bedeutung der Religion oder des Glaubens in der Gesellschaft wird in den Niederlanden trotz und alledem noch immer eine bemerkenswert lebendige öffentliche Debatte geführt. Die Frage nach Sinngebung und Weltanschauung beschäftigt auch heute noch publizierende und diskutierende Niederländer. Die Diskussion spielt sich dabei immer mehr an Orten ab, wo man es nicht erwartet hätte und wo über vielerlei (vor allem nicht-religiöse Themen) diskutiert wird. Denn auch außerhalb der speziell für christliche oder kirchliche Leser redigierten Zeitschriften oder Zeitungen wird 'Weltanschauung' zusehends ein Thema. Ähnliches gilt auch für eine ganze Reihe von Rundfunkprogrammen.

Aus der Tatsache, daß z. B. Ende 1991 'Wending', eine Zweimonatszeitschrift für Evangelium, Kultur und Gesellschaft ihr Erscheinen einstellte, darf man nicht zu rasch den Schluß ziehen, daß religiöse Themen in den Niederlanden auf abnehmendes Interesse stoßen.?

Es sind nicht nur Religionswissenschaftler, Theologen, Religionssoziologen, Religionspsychologen und in zunehmendem Maße auch Kommunikationswissenschaftler, die über Religion sprechen und schreiben, sondern auch Kolumnisten, Journalisten und Politiker. Auch in Radio, Film und Fernsehen beschäftigt man sich heute noch - oder wieder - mit Religion und kirchlichen Themen. Trotz aller Säkularisierung ist Religion offensichtlich ein wichtiger Faktor im Leben der Niederländer und in der niederländischen Gesellschaft geblieben.

So existiert Religion als die Form, in der der Glaube ein kulturelles und gesellschaftliches Gesicht erhält, in den Niederlanden noch immer und stellt einen wichtigen gesellschaftlichen Faktor dar. Sie koexistiert gewissermaßen mit den weiterhin ablaufenden Prozessen der Säkularisierung. Kirchenaustritt, Verlust von Symbolgehalten, Statusverlust der Institution Kirche, zurückgehender Kirchenbesuch und alles, was hier noch hinzuzufügen wäre, haben offensichtlich für jeden Einzelnen eine andere emotionale Bedeutung und einen anderen Erkenntniswert. 
Seit langem fragen Religionssoziologen, wie sehr Menschen sich (noch) durch traditionelle, religiöse Systeme leiten lassen. So 'entdecken' sie, daß Mitglieder einer bestimmten kirchlichen Gemeinschaft nicht ohne weiteres dasselbe glauben. Inzwischen hat sich das Arbeitsfeld der Religionssoziologie ausgedehnt auf moderne Formen der Sinngebung als 'Glaubensspuren', die außerhalb der Kirchen zu finden sind. Bei dieser sog. 'unsichtbaren Religion' geht es um Lebenseinstellungen, die außerhalb der organisierten und institutionalisierten Systeme Gestalt bekommen.

Die Religionssoziologen E. Hijmans und H. Hilhorst von der katholischtheologischen Universität Utrecht haben einen Sinngebungstyp freigelegt, den sie durch eine 'Mosaïkstruktur' gekennzeichnet sehen. ${ }^{10}$ Eigene Lebenseinstellungen und Lebensorientierungen schließen sich hier zusammen mit isolierten Elementen verschiedener Sinngebungssysteme. Diese 'Mosaikstruktur' scheint in wichtiger Weise typisch zu sein für die Lage der Religion in der heutigen Gesellschaft und für die Art und Weise, mit der Menschen mit ihr umgehen. Sie schließt ein, daß Sinngebungsprozesse in erster Linie individuell erlebt und gestaltet werden und bei einem eher schwachen vorgegebenen Ordnungsrahmen auch in hohem Maße freischwebend sein können. Zugleich stellen die beiden Forscher jedoch fest, daß der Einfluß traditioneller und moderner religiöser Ideen nicht verschwunden ist.

\section{Katholiken und die Identität von Organisationen}

1947 betrug nach Angaben des 'Sociaal en Cultureel Planbureau' die Anzahl der Konfessionslosen $17 \%$ der niederländischen Bevölkerung. Im Jahre 1991 hat sich dieser Prozentsatz mindestens verdoppelt, vielleicht sogar verdreifacht, so daß ein Drittel oder die Hälfte der niederländischen Bevölkerung sich als 'nicht kirchlich' betrachtet. Zwischen 1958 und 1987 ging die Zahl der Menschen, die sich mit der katholischen Weltanschauung identifizierte, von $33 \%$ auf $20 \%$ zurück.

Der Zeitraum von 1966 bis 1970 ist gekennzeichnet durch eine starke Ablehnung von Organisationen auf religiöser Grundlage; dies gilt sowohl für die Niederländer allgemein wie für Mitglieder der Kirchen. Zwischen 1970 und 1975 ließ sich unter Kirchenmitgliedern (aber das waren inzwischen natürlich weniger geworden) eine leichte Tendenz zur Besserung erkennen. In den 80er Jahren blieben die Meinungen im Zusammenhang mit weltanschaulicher Identifikation sowohl unter Kirchenmitgliedern wie unter den Niederländern im allgemeinen stabil. Jene, die eine starke institutionelle Identitätsbindung zeigen, sind häufig etwas älter, verfügen über eine bessere Bildung und sind wohlhabender; dies gilt sowohl für die Stadt wie auch für das flache Land.$^{11}$ Die so geschilderte soziale Gruppe bildet $\mathrm{m}$. E. auch die Zielgruppe der Bistumsblätter. Man findet sie auch unter den Freiwilligen in den Pfarrgemeinden.

Die Übereinstimmung mit dem Hintergrund der progressiven katholischen 'Acht-Mei-Beweging' ${ }^{12}$ ist frappierend. Eine Anfang 1991 durchgeführte Untersuchung unter Leitung des Theologen J. A. van der Ven von der katholischen Universität Nijmegen ergab, daß Frauen, Ältere und Intellektuelle in der 
'Acht-Mei-Beweging' im Vergleich zum durchschnittlichen Kirchenvolk überrepräsentiert sind. Zwei Drittel der Mitglieder dieser Bewegung besteht aus Frauen; $60 \%$ zählen zu den Personen mit einer höheren oder mittleren Berufsausbildung (das ist beinahe doppelt so hoch wie der Landesdurchschnitt und gut zwei mal so hoch wie der Durchschnitt bei den Katholiken). Das durchschnittliche Lebensalter liegt bei 56 Jahren; die Gruppe der Jungen fehlt bei den Anhängern der 'Acht-Mei-Beweging', während aus einer anderen Untersuchung hervorgeht, daß 10 bis $20 \%$ der Jüngeren noch zu den aktiven 'kirchlichen' Niederländern gerechnet werden dürfen. Aus der Untersuchung ging hervor, daß die Sympathisanten der 'Acht-Mei-Beweging' mit der autoritären Atmosphäre in der Kirche, mit dem Mangel an Kommunikation, mit dem Fehlen einer geteilten Verantwortung unzufrieden sind und über das Defizit an Dialog innerhalb der Kirche klagen. ${ }^{13}$

Es läßt sich fragen, ob diese gut ausgebildeten, kritischen, aktiven und motivierten Mitglieder der Kirche unter dem Schirm der 'Acht-Mei-Beweging' sie sind größtenteils Leser ihres Leitblattes 'De Bazuin' (Meinungswochenblatt für Kirche und Gesellschaft) - sich für die Bistumsblätter gewinnen ließen. Eine Untersuchung könnte als Antwort ergeben: für einige Bistumsblätter ja, für andere per se nicht.

\section{Das Paradox der Frelheit}

Die obigen Ausführungen liefern natürlich keine tiefgreifende Analyse der Situation des kirchlichen und religiösen Lebens in den Niederlanden von heute. Es ging ausschließlich darum, die Komplexität der Verhältnisse zu illustrieren. Presseorgane und Rundfunkorganisationen treten sichtbar in eine Öffentlichkeit, eine Wirklichkeit, die sehr verschiedene und kaum zu identifizierende Einfärbungen hat. Humanistische, christliche oder andere weltanschaulich gebundene Rundfunksender, Zeitungen und Zeitschriften stoßen bei ihrem Auftreten in der Öffentlichkeit auf Empfindlichkeiten und häufig auch auf Überempfindlichkeiten. Und dies nicht nur bei weltanschaulich uninteressierten Zeitgenossen, sondern gerade auch bei Individuen und Gruppen, die mit Kirche, Religion, religiöse Erziehung oder Sinngebung zu tun haben. Und dabei ist es gar nicht mehr selbstverständlich, daß es in den Niederlanden einen katholischen Sender gibt oder daß ein katholisches Nachrichtenmagazin erscheint. Die katholische Tageszeitung gehört schon längst zur Pressegeschichte. ${ }^{14}$

Typische weltanschaulich gebundene Organisationen sind in den Niederlanden politische Parteien, Erziehungseinrichtungen und Rundfunkorganisationen. Die Presseorgane werden vom 'Sociaal en Cultureel Planbureau' schon nicht mehr dazu gerechnet. Die Anhängerschaft der meisten weltanschaulich gebundenen Organisationen ist seit 1945 zahlenmäßig zurückgegangen, wie A. P. N. Nauta vom 'Sociaal en Cultureel Planbureau' im Frühjahr 1991 feststellte. ${ }^{15}$ In der Medienwelt ist der Prozentsatz von Mitgliedern konfessioneller Sender seit dem Kriege von rund $60 \%$ auf $30 \%$, also um die Hälfte, zurückgefallen. Konfessioneller Rundfunk hat also ebenso wie kirchliche Presse mit den Prozessen der allgemeinen Entkirchlichung an Umfang und Bedeutung verloren. 
Außerdem hat sich auch die Art der konfessionellen Presse- und Rundfunkorgane selbst häufig verändert. In vielen Fällen ist die Verbindung mit Religion als solcher weniger evident geworden. Außerdem haben sich manche neuen Kristallisationspunkte entwickelt; so kann man zum Beispiel gegen kommerziellen Rundfunk sein und aus diesem Grunde sich einem konfessionellen Rundfunksender zuwenden.

\section{Panta rhei oder: alles ist in Bewegung}

Wer einmal getauft ist, kann sich auch ohne große Beteiligung am kirchlichen Leben 'katholisch' fühlen. Um einmal einen Journalisten an Stelle eines Sozialwissenschaftlers zu zitieren, nehme ich ein Stück aus einer Kolumne von Beatrijs Ritsema im NRC Handelsblad. Unter der Überschrift 'Strategie' schreibt sie am 31. Januar 1991 auf der letzten Seite dieser Zeitung u. a.: 'Der Katholizismus als Volksreligion ist schon eine ganze Zeit lang auf dem Rückmarsch. Er hat sich in vielen westlichen Ländern zu einer vagen Weltanschauung ohne viele Einschränkungen und ohne viel Engagement abgeschwächt. Menschen, die sich nur bei Tauf-, Hochzeits- und Beerdigungsfeierlichkeiten in der Kirche einfinden und gelegentlich einmal eine Nachtmesse mitmachen, können sich sehr gut 'katholisch' nennen. Es ist das Paradox der Freiheit: je mehr Möglichkeiten ein Klub seinen Mitgliedern bietet, um zu tun, was ihnen gutdünkt, desto weniger Menschen sind daran interessiert, dort Mitglied zu werden oder zu bleiben.'

Der Verwaltungswissenschaftler R. in't Veld von der Erasmus-Universität in Rotterdam hat im Hinblick auf die Krise der sozialdemokratischen 'Partij van de Arbeid' sehr genau und scharfsinnig beschrieben, was mit den Niederländern im allgemeinen und der niederländischen Situation im letzten Vierteljahrhundert geschehen ist: „Unsere Vorstellungen von der Welt", so in't Veld, „über das Wünschenswerte und Mögliche, über Stellungen und Pflichten des Menschen darin sind dabei sich zu verändern, und zwar von geschlossenen, beruhigenden und sinngebenden Mustern in Richtung auf offene, reflexive und bewegliche Figuren. Man kann dies auch als eine Bewegung in Richtung auf Modernität beschreiben, das heißt funktioneller an Stelle von substantieller Ideologien. Die Eindimensionalität von Normen, Regeln und Vorlieben hat dabei keinen Platz; eine Säule bietet keinen lebenslangen Halt mehr. Wir wechseln ohne große Anstrengung unsere Mitgliedschaften. Unser Leben verläuft in einem schnellen, staccatoartigen Tempo. Wir können uns dank technologischer Entwicklungen sehr schnell bewegen und leicht miteinander kommunizieren. Unser Denken ist in erster Linie prozedural ausgerichtet, und sehr funktionell. Es ist weniger fragmentarisiert als vielmehr multidimensional. Unser Leben findet einen Zusammenhang in einer Art Tagesordnung, nicht in unveränderlichen Prinzipien. Wir sind nicht normlos, aber unsere Normen unterliegen dem Gesetz der Bewegung." 16

Lassen sich diese Überlegungen auf die Krise in einer sozialdemokratischen politischen Partei in den Niederlanden im Jahre 1991 anwenden, so gelten sie auch für die Katholiken innerhalb der niederländischen Kirchenprovinz. Weltanschaulich gebundene Medienorganisationen können sich ebensowenig 
wie sog. 'neutrale' Konkurrenten auf Dauer allgemein geltenden Regeln von Leistung und Wachstumstendenzen entziehen, weil für die weitere Existenz das Angebot dieser spezialisierten Anbieter vom Umfang der Nachfrage abhängig ist. Der weltanschauliche Pluralismus macht eine Bündelung der Kräfte schwierig, so daß innerhalb des Fächers identitätsbewußter Printmedien und Rundfunkorganisationen gelegentlich auch eine Zersplitterung auftritt. Selbst die auf der Hand liegende Zusammenarbeit zwischen der katholischen Rundfunkanstalt KRO und der protestantisch-christlichen Radiovereinigung NCRV kommt nur zögernd in Gang. Ich bin mir denn auch durchaus bewußt, daß eine Zusammenarbeit und ein Zusammengehen von Bistumsblättern ein ähnlich schwieriges Unterfangen ist. Doch können wir uns dieser Herausforderung nicht entziehen.

Die Polarität von 'progressiv' und 'konservativ' beeinflußt die Haltung gegenüber Problemen wie der menschlichen Würde, dem Mündigsein von Menschen, der Umwelt, der Arbeit und der Einschätzung ihres Wertes, gegenüber dem politischen und dem ökonomischen System, dem Denken über Euthanasie, kurzum: diese Zweiteilung beeinflußt unser ganzes Denken und Handeln. Die katholische Glaubensgemeinschaft in den Niederlanden wird außerdem noch heimgesucht durch die Gegensätze zwischen traditionellen und progressiven Gruppierungen. Auf dem Gebiet des Rundfunks hat dies schließlich nicht zur Errichtung einer traditionalistisch-katholischen Rundfunkorganisation geführt, wohl aber zu konservativen und einer harten Linie folgenden Printmedien. ${ }^{17}$ Im Raum der Bistumspresse muß man das zehnmal jährlich erscheinende Blatt De Roerom mit einer Auflage von ungefähr 2500 Exemplaren als eine progressive Alternative zum offiziellen Bistumsblatt der Diözese Den Bosch ansehen. Damit ist es seit sechs Jahren eine permanente Erinnerung an die Scheidung der Geister, die sich in der Diözese Den Bosch unmittelbar nach der letzten Bischofsernennung vollzogen hat.

\section{Das Bistumsblatt näher betrachtet}

Wie stellt sich unter den geschilderten Umständen ein Bistumsblatt dar und mit welcher Spiritualität ist es konzipiert? Mit welchen Intentionen und für wen wird es geschrieben? An diesen Fragen kann ich nicht vorbeigehen. Doch werde ich keine Formel für ein Bistumsblatt entwerfen: stattdessen werde ich am Schluß dieses Beitrages einen anderen Weg andeuten. Die gesteltte Frage schließt überdies eine Einschränkung ein, weil es in einer Diözese auch andere Wege und andere Mittel gibt, mit der sich die Kirche in die Öffentlichkeit einbringt und präsentiert.

In einem Sammelband über Medien und religiöse Weltanschauung hat Frans Oudejans 1985 denn auch nicht ohne Grund die Diözese als Nachrichtenquelle behandelt. ${ }^{18}$ Nach Oudejans hat die Erstellung und Herausgabe einer Bistumszeitung nicht die höchste Priorität, wohl aber die Förderung eines nuancierten Bildes von Kirche und Religion in den regionalen und lokalen Medien.

Gleichsam als Intermezzo ist es vielleicht ganz nützlich, einen Blick über die Grenzen zu werfen. Die Auflage der Bistumszeitungen in der Bundesrepu- 
blik Deutschland zeigt eine abnehmende Tendenz. Zwischen Ende 1985 und Ende 1990 sank die gesamte verkaufte Auflage um ungefähr 550.000 auf rund 1,5 Mio. Exemplare..$^{19}$ In Österreich konstatierte man zwischen 1974 und 1989 eine Verringerung der Gesamtauflage um $15 \%$ (von 455.700 auf 388.460 Exemplaren). ${ }^{20}$ Die Situation in beiden Ländern zeigt eine erstaunliche Übereinstimmung. Dies gilt auch für die Tatsache, daß man aufgrund wissenschaftlicher Untersuchungen schon seit vielen Jahren genau über die starken und schwachen Punkte in der redaktionellen Politik Bescheid weiß, aber die Resultate dieser Untersuchung nicht oder nur unzureichend benutzt hat, um eine Änderung in der Gestaltung der Blätter herbeizuführen.

Interessant ist auch, daß die heutigen Bistumszeitungen in Deutschland während der nationalsozialistischen Regierung entstanden sind. 1936 war nur noch ein einziges offizielles Presseorgan als Mittel zur Verkündigung in jedem Bistum zugelassen. Viele regionale Kirchenzeitungen verschwanden dadurch zugunsten einer Bistumszeitung oder eine solche wurde neu gegründet. Nicht nur in Deutschland und in Österreich, sondern auch in den Vereinigten Staaten existieren wöchentlich erscheinende Bistumszeitungen. Im übrigen ist die Bistumszeitung nach deutschem Vorbild nach einer Untersuchung von F. Oertel ${ }^{21}$ selbst in ausgesprochen katholischen Ländern keine normale Erscheinung. Er bezweifelt mit starken Argumenten die Notwendigkeit eines Presseorgans der lokalen Kirche, die sich auf das Prinzip „Organ der Ortskirche“ gründet. Durch eine Verabschiedung dieses Prinzips wäre der Weg wohl frei für überdiözesane Formen der Zusammenarbeit unter Einschluß der verschiedenen Bistümer wie auch für eine inhaltliche Einstimmung auf neue Lesergruppen.

Interessant ist auch, daß F. Oertel das Prinzip des Bistumsblattes als Forum, als Begegnungsort für die Äußerungen aller in der Kirche zur Diskussion stellt. In diesem Zusammenhang fragt er an, ob Bistumszeitungen sich nicht eher auf eine bestimmte Zielgruppe als auf die 'Masse' des Kirchenvolkes richten sollten. Wenn man die 'pluralistische Kirche' ins Kalkül zieht, müßte eigentlich eine bejahende Antwort auf der Hand liegen. Ohne einschneidende Erneuerungen und Veränderungen sieht F. Oertel die Bistumspresse in Deutschland langsam aber sicher dahinschwinden. Diese Überlegungen bedeuten auch eine Warnung für die Lage in den Niederlanden.

\section{Die Bedeutung des Pfarrbriefs}

Der Pressedienst der Diözese Rotterdam berichtet am 5. Dezember 1990, daß sich der Priesterrat des Bistums mit den Bistumsblättern beschäftigt hat. „Dem Priesterrat des Bistums Rotterdam erscheint es der Mühe wert“, so heißt es in dem Bericht, , in der Niederländischen Kirchenprovinz die Möglichkeiten der Zusammenarbeit für die Herausgabe von Bistumszeitungen zu untersuchen. Mit einer solchen Zusammenarbeit - für die Konkretisierung wird u. a. das Blatt 'Kerk en Leven' der niederländischsprachigen Bistümer in Belgien als Beispiel genannt - wird beabsichtigt, daß interessierte Diözesen sich gegenseitig mit ihrem jeweiligen Know-How helfen können, zum Beispiel bei der Planung, dem Betrieb, der Verbreitung und bei den redaktionellen Inhalten. Was die Inhalte 
anlangt, so vermutet der Priesterrat, daß z. B. ein 'pooling' guter niederländischer Autoren eine Möglichkeit wäre, um die (Glaubens-)Kommunikation zu fördern, die ja jedes Bistumsblatt notwendigerweise anstrebt.“" 22

Abgesehen von der m. E. einzigartigen Situation der Katholiken in den Bistümern in den Niederlanden kann der Vorschlag, 'Kerk en Leven' in Flandern als Vorbild zu nehmen, schon aus grundsätzlichen Erwägungen heraus nicht im Interesse der Kommunikationsstruktur der Printmedien in der niederländischen Kirchenprovinz sein. In Flandern erscheint das überdiözesane Kirchenblatt 'Kerk en Leven' ja mit einer wechselnden lokalen Pfarrseite, der Rückseite. Das ist nicht ohne Bedeutung, wie ich später noch aufzeigen möchte. Im übrigen behandelt das Blatt Themen aus der Weltkirche und aus der flämischen Kirchengemeinschaft, mit Wechselseiten für jedes Bistum.

'Kerk en Leven' wurde 1946 als 'Kerkelijk Leven' gegründet. Es sollte um das Angebot allgemeiner Information gehen, in Kombination mit einer auf die lokale Gemeinschaft ausgerichteten kirchlich religiösen Berichterstattung. „Unter dem Anschein, jeder einzelnen Pfarrei ein eigenes Pfarrblatt zu liefern, erhielten die Abonnenten auch allgemeine Informationen“, so Marcel de Roover über die Taktik, die man anwandte, um das Blatt bei den Pfarrern akzeptabel zu machen. ${ }^{23}$ Von Anfang an war 'Kerkelijk Leven' also mehr als ein Pfarrblatt im Sinne eines Kommunikationsmediums zwischen Priestern und Pfarrangehörigen. Später war es das Organ von und für die Gemeinschaft der Pfarreien in allen Gliederungen. In Flandern hat man seit 1941 systematisch daran gearbeitet, die Pfarrblätter als selbständige Ausgaben zum Verschwinden zu bringen.

Die Auflage von 'Kerk en Leven' bewegt sich um die 770.000 Exemplare (mit einer Spitze über 800.000 gegen Ende der 80er Jahre). Das ist eine sehr eindrucksvolle Zahl. Unter neuer redaktioneller und kaufmännischer Leitung hat sich das Blatt seit einigen Jahren einem redaktionellen und technischen Erneuerungsprozeß unterzogen, aber die Formel einer allgemeinen Kirchenzeitung mit einer Wechselseite für Pfarrneuigkeiten ist erhalten geblieben. Mir fällt dabei auf, daß diese Seite stark an die früheren niederländischen kirchlichen Nachrichten erinnert, die sowohl in den regionalen kirchlichen Tageszeitungen wie auch in den früheren Bistumszeitungen erschienen. Ich gebe der Pfarrblattkultur den Vorzug, wie sie sich nach dem Verschwinden der regionalen Presseorgane in den 60er Jahren in den Niederlanden entwickelt hat. Die kirchlichen Nachrichten alten Stils trifft man noch immer in Teilen der Niederlande, sowohl in Nachrichtenblättern wie auch in Haus-zu-Haus-Zeitungen, die kostenlos verteilt werden. Wie schon gesagt, handelt es sich bei der Rückseite von 'Kerk en Leven' in Flandern um nicht viel mehr als eine Dienstleistungsseite mit kirchlichen Berichten.

Die Hauptaufgaben einer Pfarrei finden $m$. E. ihren publizistischen Ort am besten in Pfarrblättern. Als die Hauptaufgaben einer Pfarrei gelten in jedem Fall:

- die Verkündigung (Lehren - Katechese);

- Heiligung (Feier - Liturgie);

- Caritas (Werke - Diakonie). 
Nach Angaben des katholischen Medienzentrums in Zeist haben $82 \%$ der fast 1800 Pfarrgemeinden in den Niederlanden ein eigenes Blatt, weitere $13 \%$ kennen ein gemeinsam produziertes Organ (überpfarrlich, zum Beispiel im Fall einer Distriktpfarrei); nur $5 \%$ benutzen ausschließlich redaktionellen Raum in einer lokalen oder regionalen Zeitung.

Im Hinblick auf das Verhältnis der Bistumszeitung zu den Pfarrgemeinden muß noch folgendes angemerkt werden. 'Aartsbisdom Nieuws', 1990 umbenannt in 'Op Tocht', nennt sich im Untertitel 'Informationsbulletin für die Pfarreien im Erzbistum Utrecht'. 'De Roerom' ist gleichfalls auf die Pfarrei als christliche Glaubensgemeinschaft gerichtet. Das im 7. Jahrgang erscheinende Blatt 'De Roerom' nennt sich 'Nieuws-, service- en communicatieblad ten dienste van de parochies in het Bossche bisdom, om een handje te helpen de gang erin te houden'. Im Lichte des II. Vatikanischen Konzils ist die Ortsgemeinschaft der Gläubigen, die 'Basisgemeinschaft', als das Herz der Kirche anzusehen.

Das Pfarrblatt ist das Hauptkommunikationsmedium der Basis der Niederländischen Kirche. Es gehört in die bunte Gesellschaft des publizistischen Fußvolkes der ohne Gewinnabsichten verbreiteten Zeitschriften von freiwilligen Organisationen. Das Interesse der Zeitungsmacher liegt darin, Engagement der Leser bei der Realisierung der Ziele der Pfarrei zu wecken. In der redaktionellen Formel wird idealerweise auch auf die verschiedenen Kirchenvorstellungen und Auffassungen über die gewünschte Richtung der Pfarreien Rücksicht genommen. Das Pfarrblatt basiert auf einem Typ der Kommunikation, der kennzeichnend ist für den Dialog von Menschen, die zusammen auf der Suche nach Sinngebung sind, darüber gelegentlich berichten wollen und sich bei diesem suchenden Vorantasten das Religiöse und die Glaubensgemeinschaft vor Augen halten.

In einer Mischform von Servicerubriken und Beiträgen, die auf Besinnung oder Aktion gerichtet sind, kann das Pfarrblatt eine Podiumsfunktion erfüllen. Auf diese Weise läßt es die Verschiedenheit und Breite an Kirchen- und Menschenbildern mit ihren Gefühlen für Kirche und Pfarrei zu ihrem Recht kommen. Mit ein und derselben Ausgabe muß ja ein sehr heterogen zusammengesetztes Leserpublikum angesprochen werden. Derjenige, der ein Pfarrblatt in die Hand nimmt, befindet sich in einem bestimmten Abstand von der Pfarrei, der Redaktion und den in dem Blatt behandelten Themen. Ob dieser Abstand überbrückt werden kann, hängt auch von dem individuellen Leser ab. Die Redaktion kann nur ihr bestes tun, um so viele Barrieren zum avisierten Leser wie möglich zu überwinden. Kirchliche und kirchlich orientierte Blätter haben in hohem Maße gerade für den kirchlich interessierten und kirchlich gebundenen Menschen eine Funktion. Das gilt auch und gerade für das Pfarrblatt.

Der Pastoraltheologe Ernest Henau sieht die Pfarrei als die geeignetste Organisationsform an, um eine Gemeinschaft zu bilden und Menschen Dienstleistungen anzubieten; auch jenen, die nicht so intensiv mit der Kirche mitleben. Gemeinschaftsbildung heißt zugleich, daß man Polarisation vermeidet und gewachsene Gegensätze überbrückt. Kleine Glaubensgemeinschaften am Rand der Pfarrstruktur dürften entsprechend dieser Auffassung über das Funktionie- 
ren einer Pfarrei nicht (weiter) an den Rand gedrückt werden. Anerkennt man das Recht auf eine freie(re) Meinung, dann dürfte der Kontakt zwischen den eher traditionell ausgerichteten Pfarreien und z. B. den Basisgemeinden doch hergestellt und beibehalten werden. ${ }^{24}$

Wegen dieser und anderer Gründe sollte das Pfarrblatt als Kommunikationsmedium von und für die Pfarrei verstärkt werden. ${ }^{25}$ Dies geschieht jetzt zielgerichtet und planmäßig durch Aufbaukurse für Pfarrblattredakteure und redakteurinnen. Es handelt sich dabei um ein gemeinsames Projekt des katholischen Medienzentrums, des katholischen Rundfunks (KRO), der Pressedienste der niederländischen Bistümer und der diözesanen Zentren für die Pastoral. ${ }^{26}$

\section{Hin zu Zusammenarbeit und Verschmelzung}

Daß die traditionellen Bistumsblätter der vorkonziliaren Zeit ihren Charakter geändert haben oder sogar eingestellt wurden, läßt sich im Lichte der Veränderungen nach dem II. Vatikanischen Konzil verstehen. Schwerer verständlich ist jedoch, warum damals kein Periodikum von allen Bistümern gemeinsam herausgegeben wurde. In den Jahren 1968 und 1969 besaßen die drei südlichen Diözesen Den Bosch, Roermond und Breda für eine kurze Zeit ein gemeinsames Bistumsblatt. Danach haben diese Diözesen sich wieder für ein je eigenes Blatt entschieden. Es müßte näher untersucht werden, warum es nicht zu einer dauerhaften Zusammenarbeit zwischen einigen oder allen Bistümern in Sachen ihrer Bistumszeitungen gekommen ist. Der Polarisationsprozeß zwischen traditionellen und progressiven katholischen Gruppen hat damals ein Ventil in Zeitschriften gefunden, die keine Beziehungen zu den Bistümern hatten. Schon vor langer Zeit scheint man eine Chance verpaßt zu haben, ein gemeinsames Wochenblatt der niederländischen Bistümer oder der niederländischen Kirchenprovinz zu gründen.

Die Schaffung von Blättern oder Bulletins auf Dekanatsebene zeigt diese Schatten nicht, obwohl die Redaktionen dieser Blätter sich ausdrücklich auf die Funktion von Kaderblättern für die im Dekanat oder in den Pfarreien aktiven freiwilligen Mitarbeiter beschränken. ${ }^{27}$ Wenn sich ein solches Blatt nicht ausdrücklich auf das Gebiet von Pfarrblättern begibt, dann kann ein solches Periodikum von und für ein Dekanat den Charakter eines Informationsbriefes oder eines Bulletins für eine klar umschriebene Zielgruppe haben.

Da jetzt das Bistumsblatt in der niederländischen Kirchenprovinz in manigfacher Form in jeder Diözese wieder besteht, ergibt sich die Frage, wie die Bistumspresse am effektivsten und wirksamsten ihren spezifischen Auftrag erfüllen kann. Dazu möchte ich versuchsweise 10 Thesen über die Zukunft der Bistumszeitung vorlegen. Dies bietet mir zugleich die Gelegenheit, aus den vorhergehenden Darlegungen Schlußfolgerungen zu ziehen.

\section{Schlußfolgerungen und Thesen}

1. Die Lage der katholischen Glaubenæigemeinschaft in den Niederlanden ist zu $99 \%$ das Ergebnis jahrhundertelanger historischer Prozesse und nur zu $1 \%$ 
das von gegenwärtigen Faktoren. Beide Kraftfelder haben eine als ziemlich einzigartig zu bezeichnende Struktur und Mentalität des katholischen Lebens in den Niederlanden hervorgebracht. Dies hat auch zur Folge, daß die Förderung der Kommunikation zwischen Bischöfen und Gläubigen, aber auch zwischen den verschiedenen Typen und Gruppen von Katholiken untereinander, in ganz eigener Weise angepackt werden muß. Ausländische Kommunikationsmodelle bieten für die niederländischen Verhältnisse keine Lösung.

2. Da in den letzten 20 Jahren die Verbindungen der Bistümer zur Kirche als Weltkirche einerseits und zu den örtlichen Glaubensgemeinschaften oder zu den 'lokalen und regionalen' Basisgemeinden andererseits, flexiblere Formen angenommen haben, haben die Diözesen bei jenen Menschen, die weniger aktiv am kirchlichen Leben teilnehmen, immer mehr an Einfluß verloren. Durch bestimmte kirchenpolitische Entwicklungen, z. B. im Zusammenhang mit der Ernennung bestimmter Bischöfe, wie auch durch die größere Bedeutung von Pfarreien und Basisgemeinden als Orten des Glaubens ist das Bistum in der gleichen Zeit auch für die aktiveren Katholiken aus dem Blickfeld geraten. Was für das Bistum als Organisation gilt, braucht jedoch nicht für die Bischöfe und ihre nächsten Mitarbeiter zu gelten. Dies umso mehr, als sie sich um den persönlichen Kontakt mit'den Menschen in ihrem Bistum bemühen.

3. In den Niederlanden herrscht immer noch Interesse für das kirchliche und religiöse Leben. Viele Menschen zeigen eine bestimmte Affinität für religiöse Themen und Motive. Die gesellschaftlichen Säkularisierungsprozesse haben nicht dazu geführt, daß das Interesse der Massenmedien (Zeitungen, Zeitschriften, Radio und Fernsehen) an kirchlichen, religiösen und mit Sinngebung zusammenhängenden Themen nachgelassen hat. Als Folge von Veränderungen im Journalismus scheint allerdings die Aufmerksamkeit stärker auf fragmentarisierte und zufällige Ereignisse gerichtet zu sein. Ein Magazin von und für die katholische Glaubensgemeinschaft in den Niederlanden hat dann nicht als Ersatz für einen solchen Journalismus, sondern als Ergänzung dazu einen Sinn.

4. Der kirchenrechtliche Status des Bischofs als 'örtlicher Ordinarius' steht der Herausgabe eines nationalen Magazins im Dienste der Kommunikation über Angelegenheiten der Kirche und der Gesellschaft nicht im Wege. Dadurch daß es keine Kirchensteuer in der niederländischen katholischen Glaubensgemeinschaft gibt, hat sie den Charakter einer freiwilligen Kirche. Bei allen Vorteilen besteht natürlich der Nachteil, daß die finanzielle Basis für die je für sich erscheinenden Bistumsblätter im Durchschnitt schwach ist. Die finanzielle Situation unterscheidet sich von Bistum zu Bistum auf Grund struktureller und zufälliger Gegebenheiten. Beides hat Folgen für die Unterschiede zwischen den bestehenden Bistumsblättern mit Bezug auf Erscheinungsfrequenz, typographische Aufmachung und die Qualität des redaktionellen Teils.

5. Eine freiwillige redaktionelle Zusammenarbeit zwischen den bestehenden Bistumsblättern ist $\mathrm{m}$. E. ein zu langer und zu riskanter Weg, um schließlich zur Herausgabe eines Blattes zu kommen. Eine sich allmählich entfaltende Zusammenarbeit verlangt zu viel Energie der sowieso knappen 'manpower' und bringt erst nach einem Zeitraum von fünf bis zehn Jahren die jetzt schon not- 
wendigen Einsparungen. Eine Maßstabvergrößerung muß zu einer Bündelung finanzieller Mittel führen und zu einer optimalen Ausnutzung der knappen journalistischen Talente auf dem Gebiet von Kirche und Gesellschaft. Außerdem würde eine einschneidende Bündelung von Kräften, Mitteln und Talenten die Glaubwürdigkeit dieser spezifischen Form des Journalismus vergrößern.

6. Um die Kommunikation des Volkes Gottes in den Niederlanden mit der Weltkirche und mit den örtlichen Glaubensgemeinschaften und Basisgemeinden zu fördern, wäre es angeraten, kompromißlos für ein Wochenblatt einzutreten, das nicht von den Bischöfen geleitet und beherrscht wird. Um dorthin zu gelangen, wäre der folgende Weg denkbar: Die katholische Bischofskonferenz beauftragt die bischöfliche Kommission für die soziale Kommunikation, in enger Zusammenarbeit mit dem Kollegium der Pressechefs ein Konzept und ein Redaktionsstatut auszuarbeiten. Fachleute aus dem Verlagsbereich erarbeiten im Zusammenspiel mit den Ökonomen der Diözesen einen Verlagsplan. Wenn Konzept, Redaktionsstatut und Verlagsplan von der Bischofskonferenz gutgeheißen sind, ernennt dieses Organ den Herausgeber und den Chefredakteur.

7. Nach Form und Inhalt wäre dieses zu gründende Blatt ein Magazin, das wöchentlich erscheint und ausgerichtet ist auf alle, die sich für die Entwicklungen in Kirche und Gesellschaft interessieren, wobei die katholische Glaubensgemeinschaft besondere Aufmerksamkeit zu erfahren hätte. Als Beispiel könnte das seit März 1990 erscheinende Magazin 'Kerk' dienen, das ursprünglich wöchentlich erschien und im September 1991 auf monatliches Erscheinen umgestellt wurde. Das Magazin 'Kerk' in seiner neuen Form, ausgegeben vom christlichen Pressebüro Internieuws (INS) ${ }^{28}$, informiert über Nachrichten, Hintergründe und Entwicklungen in der christlichen Welt. Es besitzt einen reformatorischen Charakter. Im Licht der Ökumene könnte eine redaktionelle und wirtschaftliche Zusammenarbeit mit dem Magazin Kerk angestrebt werden.

8. Die für die interne und externe Kommunikation verantwortlichen Funktionäre der Bistümer könnten sich nach der Gründung des neuen Magazins (noch) zielstrebiger für ihre Informationsaufgaben einsetzen. Die neue Zeitschrift bliebe dabei eine ihrer Publikationsmöglichkeiten. Falls finanziell vertretbar, könnte den Informationsverantwortlichen der Bistümer jeweils eine Seite oder vielleicht sogar ein Bogen anvertraut werden. Mitteilungen und Berichte aus den Bistümern, die nicht von nationalem Interesse sind, könnten so einen Ort in dem neuen Blatt erhalten. Im übrigen gilt, daß die in den 90er Jahren entwickelten lokalen Rundfunksender (zur Zeit sind es etwa 300) für die Unterrichtung aus den Bistümern neue Perspektiven bieten.

9. Journalismus ist mehr als das Aufspüren, Bearbeiten und Weitergeben von Nachrichten. Die Nachrichtenanalyse und die Hintergrundinformation sind hier ein wesentlicher Bestandteil, ebenso wie die Meinungsbildung und die Unterhaltung. Ein Journalist, der dies alles oder nur einen Teil davon betreibt, arbeitet bewußt oder unbewußt mit am Prozeß der Sinngebung und Deutung. Er liefert Interpretationsrahmen. Wenn man diese Tatsache anerkennt, entsteht Raum für einen neuen Journalismus im Bezug auf Religion und Weltanschauung (und in geringerem Maße mit Bezug auf sog. "kirchliches Reden“). 
10. Die Gesamtauflage der Bistumsblätter und von „1-2-1“, dem Informationsbulletin des Sekretariates der katholischen Kirche der Niederlande kann auf etwa 145.000 Exemplare geschätzt werden. ${ }^{29}$ Diese Auflage bietet keine Garantie für den Erfolg eines neuen Wochenblattes, stellt aber einen guten Ausgangspunkt dar, um einen gemeinsamen Schritt in die Zukunft zu wagen. Bleibender Erfolg wird in nicht geringem Maße abhängig sein von der Frage, ob ausreichend professionelles Talent in der jüngeren Journalistengeneration vorhanden sein wird. Weiter werden die Bischöfe zu erkennen geben müssen, daß sie in diesem neu zu gründenden Magazin einen kritischen und unabhängigen Journalismus schätzen.

\section{Anmerkungen}

1 Daß dieser Beitrag nur von den katholischen Printmedien handelt, hat vor allem einen pragmatischen Grund: die Untersuchung hätte sonst eine viel breitere Grundlage bekommen müssen. Vielleicht fühlt sich ein Fachmann auf dem Gebiet der nichtkatholischen kirchlichen Presse angesprochen, eine Fortsetzung dieses Artikels zu schreiben. Dies wäre in jedem Fall sehr zu begrüßen.

In den meisten reformatorischen Gemeinschaften ist die über die Jahre hinweg gewachsene Struktur der kirchlichen Blätter erhalten geblieben. Initiativen, die darauf gerichtet sind, gemeinsame Periodika herauszugeben, werden dadurch nicht einfacher. Bei 'Samen op Weg'-Gemeinden ( hervormd" und "gereformeerd") ist die kombinierte.Herausgabe eines Gemeindeblattes wohl gelungen. Auch kann man die exemplarisch zu nennende Gründung des Magazins 'Kerk' (Kirche) als einen Versuch sehen, auf ökumenischer Basis zu einem neuen Anfang kirchlicher Pressearbeit zu kommen.

2 Vgl. dazu M. Schmolke, Braucht die Kirche eine neue Medienpolitik?, in: Communicatio Socialis 23 (1990), 197-206.

3 So ein Bericht des 'Algemeen Nederlands Persbureau' (ANP) in der Tageszeitung Trouw vom 5. September 1991.

4 Johann Baptist Metz, 'Kirchliche Kommunikationskultur. Überlegungen zur Kirche in der Welt der Massenmedien', in: Communicatio Socialis 24 (1991), 247-267.

5 Hella Tompert gab diesen Ehrentitel in ihrem Vorwort zu 'Kirche und Kommunikation. Zur bleibenden Aktualität von Communio et progressio', in: Medienpraxis, Grundlagen 6, Sonderheft zum Welttag der Sozialen Kommunikationsmittel 1991, 34. Darin auch Beiträge von Bernhard Laux und Hans Gasper.

6 Bisdomblad vom 11. Januar 1991, 3.

7 Die Einführungen und der Schlußtext der Beratung sind enthalten im Schlußbericht 'Christenen en de media', Publikation Nr. 23 des Interdiocesaan Pastoraal Beraad (Guimardstraat 1, B-1040 Brussel).

8 L. de HOVRE; 'Zorgen en bekommernissen vanuit het kerkelijk beleid', ebd., 21-30, hier 26-29.

9 Wending, gegründet 1946, als eine progressive, protestantisch-christliche Zeitschrift mit einer für kirchlich Engagierte ungewohnten, nämlich sozialdemokratischen Gesellschaftssicht, hatte 1991 nur noch 1400 Abonnenten. Das Blatt hatte in den letzten Jahrzehnten seiner Existenz eine ausgeprägt ökumenische Richtung bekommen. Die Zweiwochenzeitschrift 'Evangelisch Commentaar' hat am 1. April 1991 zu bestehen aufgehört. Das Blatt, eine Fortsetzung des 'Gereformeerd Weekblad', hatte im ersten Jahr seines Erscheinens (1982) 3.000 und bei seinem Ende noch 1600 Abonnenten. Die Redaktion wollte mit 'Evangelisch Commentaar' einerseits die Diskussion über Samen op Weg der 'Nederlandse Hervormde Kerk' und der 'Gereformeerde Kerken' in den Niederlanden stimulieren und andererseits Gemeindemitglieder heranbilden. 
10 E. Hijmans und $H$. Hilhorst, 'Hedendaagse vormen van zingeving'. De 'onzichtbare' religie van de Nederlander, in: Osmund Schreuder und Leo van Snippenburg (Hg.), Religie in de nederlandse samenleving. De vergeten factor, Baars 1990, 137-164.

11 Vgl. dazu einen Vortrag über den Glauben und die Wahl identitätsgebundener Organisationen, die A. P. N. Nauta vom 'Sociaal en Cultureel Planbureau' (SCP) am 22. März 1991 gehalten hat, abgedruckt in SCP Weekbericht 1991, Nr. 1, 1-3.

12 In der 'Acht-Mei-Beweging' arbeiten etwa 100 Organisationen zusammen, um von einem gesellschaftskritischen und kirchenkritischen Blickwinkel aus den Geist des II. Vatikanischen Konzils in den Niederlanden am Leben zu erhalten. Jedes Jahr findet eine Kundgebung statt; dieser 'Acht-Mei-Dag' wurde 1991 zum 7. Mal gehalten und von etwa 10.000 Sympathisanten besucht. Ein mehr konservatives Gegenstück zur 'Acht- Mei-Beweging' ist das 'Contact Rooms-Katholieken' (CRK), das im Gegensatz zur 'Acht-Mei-Beweging' gute Kontakte zu den Bischöfen unterhält. Diese Gruppe hielt 1991 ihren 6. nationalen Tag ab. Kann man das 'weekblad voor kerk en samenleving' De Bazuin als die Stimme der 'Acht-Mei-Beweging' betrachten, dann ist das zwei Mal pro Woche erscheinende Katholiek Nieuwsblad das Organ der Anhänger vom CRK.

13 So die Zusammenfassung dieser Untersuchungsergebnisse in der Tageszeitung Trouw vom 23. Februar 1991.

14 Siehe in diesem Zusammenhang den Band: Mechteld de Coo Wijgerinck, Otto S. Lankhorst en Jan Roes ( $\mathrm{Hg}$.), De gezegende pers. Aspecten van de katholieke persgeschiedenis in Nederland tijdens de 19de en20ste eeuw, Zeist 1989.

$15 \mathrm{Vgl}$. Anm. 11.

16 Roel in't Veld, 'PvdA heeft selectie van bestuurlijk talent sterk verwaarloosd', in: de Volkskrant vom 19. Juni 1991 (Open Forum).

17 Siehe in diesem Zusammenhang Joan Hemels, 'Piet Derksen und seine Medienarbeit: Stein der Weisen oder Stein des Anstoßes?', in: Communicatio Socialis 24 (1991), 6379; sowie ders., Veränderungen in der katholischen Publizistik der Niederlande, in: ebd., 197-199.

18 Frans Oudejans, 'Een bisdom als nieuwsbron', in: Joan Hemels en Henk Hoekstra ( $\mathrm{Hg}$.), Media en religieuze communicatie. Een uitdaging aan de christelijke geloofsgemeenschap, Hilversum 1985, 213-219.

19 Quelle: Informationen (Brief der Gesellschaft katholischer Publizisten Deutschland e. V.), Nr. 6, Juni 1991, 3.

20 Vgl. Norbert P. Feldinger, 'Kirchenzeitungsleser: Profil und Bedürfnisse. Eine Sekundäranalyse', in: Communicatio Socialis 23 (1990), 224-235.

21 Ferdinand Oertel, 'Die Krise der Bistumspresse 1989. Bestandsaufnahme und Zukunftsüberlegungen', in: Communicatio Socialis 22 (1989), 31-43. Diese Ausgabe ist hauptsächlich der kirchlichen Presse in deutschsprachigen Ländern gewidmet. Was Österreich betrifft, so werden die Ergebnisse einer Untersuchung publiziert, die sich mit Lesern beschäftigt, die ihr Abonnement des Bistumsblattes der Erzdiözese Salzburg gekündigt hatten. Aus den Stichproben der Untersuchten (400 Personeh) ging hervor, daß 178 gestorben waren, wodurch schon ein wichtiger Grund für die Kündigung ans Licht kam. Vgl. Franz Rest, 'Die Abbesteller des Salzburger „Rupertusblatt“, ebd., 61-75.

22 Diesen Text verdanke ich dem Pressechef des Bistums Rotterdam, Herrn H. Heruer.

23 Die Entwicklung und die augenblickliche Situation von 'Kerk en Leven' werden gründlich untersucht in Marcel de Roover, 'Kerk en Leven. Eeen „apart“ medium', in: Communicatie 18 (1988), 13-26, hier 15.

24 Siehe in diesem Zusammenhang den Bericht über einen Kongreß über die Pfarrei von Jos Berkelmans, 'Parochies voor vandaag en morgen', in: Bisdomblad vom 9. August $1991,14$.

25 Für die Einbettung des Pfarrblattes in den größeren Zusammenhang der in- und externen Kommunikation einer Pfarrei, siehe den Beitrag von Ben Spekman, 'Interne en externe communicatie van een parochie', in: Hemels en Hoekstra (Hg.), Religieuze communicatie, 226-231. 
$26 \mathrm{Vgl}$. Marianna van den Broek, Parochieblad, hoe doe je dat? Praktische handleiding voor redactiewerk, Zeist (Katholiek Mediacentrum) 1990, mit den dazugehörigen Ausgaben Harry van der Wouw (Endredakteur), Marianna van den Broek e. a. (Hg.), Parochieblad,hoe doe je dat? Een cursus redactiewerk in zeven delen. Wegwijzer voor cursusdeelnemers, Zeist (Katholiek Mediacentrum) 1990, sowie auch 'Parochieblad', hoe doe je dat? Een cursus redactiewerk in zeven delen. Handleiding voor cursusbegeleiders, Zeist (Katholiek Mediacentrum) 1990.

27 Siehe in diesem Zusammenhang Lambert van Gelder, 'Propaganda en voorlichting voor een dekenaat', in: Hemels en Hoekstra ( $\mathrm{Hg}$.), Religieuze communicatie, 220225.

28 Aus einem Bericht der Tageszeitung Trouw vom 1. Juli 1991 läßt sich schließen, daß zusammen mit der Änderung der Erscheinungsfrequenz des Magazins Kerk - Beginn September 1991 - auch eine redaktionelle Trennung stattgefunden hat zwischen dem christlichen Pressebureau Internieuws (INS) und dem Magazin Kerk. Die Redaktionen beider Organisationen sind seither getrennt. INS/Kerk muß innerhalb von drei Jahren ein rentables Tochterunternehmen des Nedag Verlags werden. Das Mutterunternehmen Nedag ist Herausgeber vom 'vrijgemaakt gereformeerde' Nederlands Dagblad in Barneveld ('vrijgemaakt gereformeerd' = Abspaltung innerhalb der 'gereformeerde' Kirche, ca. 1944). Nach De Bazuin vom 30. August 1991, 2, haben Ergebnisse einer Leseranalyse erbracht, daß nur $3 \%$ der Leser von Kerk Katholiken sind.

29 Es geht um die folgenden Titel:

Titel

Bistum

Jahrgang

Erscheinungs(1991) frequenz

$\begin{array}{llrlr}\text { Bisdomblad } & \text { Groningen } & 24 & \text { 11 Mal pro Jahr } & 2.000 \\ \text { Op Tocht } & \text { Erzbistum Utrecht } & 3 & 11 \text { Mal pro Jahr } & 8.000 \\ \text { Samen Kerk } & \text { Haarlem } & 16 & 10 \text { Mal pro Jahr } & 48.000 \\ \text { Tussenbeide } & \text { Rotterdam } & 18 & 8 \text { Mal pro Jahr } & 56.000 \\ \text { Bisdomblad } & \text { Breda } & 5 & 10 \text { Mal pro Jahr } & 4.000 \\ \text { Bisdomblad } & \text { 's Hertogenbosch } & 69 & \text { wöchentlich } & 10.000 \\ \text { De Sleutel } & \text { Roermond } & 19 & 2 \text { Mal pro Monat } & 10.000\end{array}$

Ferner: 1-2-1 Informationsbulletin des Sekretariates der Katholischen Kirche der Niederlande (erscheint $20 \mathrm{Mal}$ pro Jahr in einer Auflage von 7.000 Exemplaren). 


\section{SUMMARY}

For the structure of communication between Catholic communities, since Vatican II parish letters are of great importance. In the Netherlands, they are at the basis of the Church a unique medium for supporting the main tasks of a parish (to preach, to lead to salvation and to do the works of welfare). Diocesan papers, however, in the last years did not succeed, in providing the majority of believers with the necessary informations. In order to achieve a freater and better communication, the author pleads for giving up traditional diocesan papers run and dominated by bishops; instead, manpower and financies should be installed in a new independent weekly magazin for Church and society. Official informations of the dioceses could be integrated on variable pages. In the authors view keeping up traditional diocesan papers would increase the loss of credibility of the religious contents and not lead to the necessary economical recovery.

\section{RÉSUMÉ}

Depuis le concile Vatican II, la lettre paroissiale remlit une fonction particulière dans la structure de communication de la communauté catholique. Moyen de communication à la base de la province ecclésiastique néerlandaise, elle représente un instrument inégalé pour soutenir le p̀rincipales missions de la paroisse, soit prêcher, sanctifier et pratiquer l' assistance caritative.

Les journaux des èvêchés par contre ne sont pas parvenus, au cours des dix dernières années, à satisfaire le besoins d' information particuliers émnanant d' une assez grande partie du groupe-cible. L' auteur, pour des raisons d' efficacité et de rendement, plaide en faveur d' une suppression de ces organes de presse dirigés et dominés par les évêques. Il serait plus judicieux $d^{\prime}$ investir et ressources humaines et moyens financiers en créant un magazine hebdomadaire indépendant pour l' Eglise et la société. Le rapports officiels des différents évêchés pourraient, au moyen de pages ou de feuillets mobiles distincts, être intégrés dans cette nouvelle publication. De l' auteur, le fait de poursuivre ou de conserver les journaux épiscopaux dans leur forme actuelle rend leur contenu relegieux de moins crédible et n' ouvre aucune perspective d'assainissement économique.

\section{RESUMEN}

En la estructura de comunicación de la comunidad de fe católica, la carta pastoral de la parroquia cumple una función especial desde el Concilio Vaticano II. Como midio de comunicación con la base de la provincia eclesial holandesa es un medio único de apoyo a las tareas principales de la parroquia (predicar, santificar y caritas).

Por el contrario, los periódicos episcopales en los últimos decenios no han logrado satisfacer las necesidades de información especiales de una gran parte de los grupos interesados. El autor, por razones de efectividad y capacidad productora, aboga por la desaparición de estos órganos de prensa dirigidos y dominados por los obispos. De esta manera se podrían ultilizar de mejor, tanto el potencial humano como los medios financieros en la creación de una publicación semanal dedicada a la iglesia y a la sociedad, que fuera independiente. Los informes oficiales de los diferentes episcopados podrían ser integrados en la nueva revista con ayuda de páginas o de cuadernillos alternados. Al juicio del autor, la continuación o mantención de las hojas episcopales significa una creciente pérdida de credibilidad del contenido religioso, sin espectativas de un saneamiento económico. 